\title{
The Influence of Additional Surface on Force Platform's Ground Reaction Force Data During Walking and Running
}

\author{
Shariman Ismadi Ismail ${ }^{1,2, ~ *}$, Hiroyuki Nunome ${ }^{2}$, Fatin Farhana Marzuki ${ }^{1}$, Izzat Su'aidi ${ }^{1}$ \\ ${ }^{1}$ Faculty of Sports Science and Recreation, Universiti Teknologi MARA, Shah Alam, Malaysia \\ ${ }^{2}$ Faculty of Sports and Health Science, Fukuoka University, Fukuoka, Japan
}

Email address:

shariman_ismadi@salam.uitm.edu.my(S. I. Ismail),shariman_2000@yahoo.com(S. I. Ismail)

${ }^{*}$ Corresponding author

\section{To cite this article:}

Shariman Ismadi Ismail, Hiroyuki Nunome, Fatin Farhana Marzuki, Izzat Su'aidi. The Influence of Additional Surface on Force Platform's Ground Reaction Force Data During Walking and Running. American Journal of Sports Science. Vol. 6, No. 3, 2018, pp. 78-82. doi: 10.11648/j.ajss.20180603.12

Received: April 2, 2018; Accepted: April 20, 2018; Published: May 15, 2018

\begin{abstract}
This study compares the vertical ground reaction force exerted from walking and running movement on two different surfaces of a force platform. Five skilled male futsal players were recruited to perform the walking and running tasks over the force platform. In the first setting, the players moved directly in contact with the force platform's bare surface while in the second setting, the players performed the same tasks on the force platform covered with a typical futsal pitch surface material. The force from the peak heel and peak forefoot strikes were recorded and used for further analysis. A paired t-test was conducted for comparison and the results indicated that there are no significant differences between the two force platform conditions in terms of the magnitude of peak heel strike and peak forefoot strike forces during walking and running. Results also showed that there is a significant difference $(\mathrm{p}<0.05)$ on the ratio of the heel and forefoot strike peak force during walking task (1.05 in force platform bare surface, 0.99 in force platform + futsal court surface). While the data obtained in this study suggests that an additional surface on the force plate has no significant effect on the magnitudes of vertical ground reaction force data, there were indeed some changes that occurred on the heel/forefoot strike force ratio in walking task. The result might suggest that an additional surface (futsal court material) on the force platform has some impacts on the movement pattern of the foot during walking task due to the different conditions of the shoe's outsole-flooring surface interaction.
\end{abstract}

Keywords: Force Plate, Ground Reaction Force, Futsal Court, Movement Pattern

\section{Introduction}

The study of human locomotion has been a subject of interest for such a long time since $300-500 \mathrm{BC}$ to be exact [1]. Historically, the interest upon the knowledge of human locomotion varies by many reasons with different motivations [2] and probably differs based on different scientific community focus research area. During locomotion, force is being applied to the ground, creating a counteracted reaction forces commonly cited as the ground reaction force (GRF). Studies of the ground reaction force on human locomotion has been conducted largely in the past, particularly related to walking and running [3-6].

Past study has shown that humans adjust their leg's stiffness when running on different surfaces to be able to maintain the running mechanics [7]. This involves alteration on the ground reaction force and the ground contact time to accommodate different surface interactions. In biomechanical study, force platform is commonly used to measure the ground reaction force resulting from the movement of humans or animals [8]. It is widely accepted that the source of the ground reaction force value measured by the force platform is related towards the body's center of mass. Therefore, there were suggestions that only alterations that are directly or indirectly related to the body's center of mass would be able to influence changes on the ground reaction force [9] 
and it does not influence by footwear. In contrast, there were also studies that demonstrated the influence of type of surfaces and footwear towards altering the ground reaction force properties [10]. The interaction between two surfaces (floor and footwear) during movement potentially influences the data of the ground reaction force.

In sports biomechanics study, researchers would normally install sports surface material onto the force platform for the purpose of analyzing kinetics variables. This creates analysis environment that is similar to the real situation, thus aims to obtain a representable kinetics data from the laboratory-based experiment. However, there are situations where the exact identical surface of interest to be analyzed was unable to be replicated or that it required massive work to recreate within laboratory testing environment. For example, a study regarding the interaction between road marathon runner's shoe outsole-road surface or the interaction between beach soccer player's foot-beach surface would be very difficult to be conducted within a laboratory-based force plate set-up. Therefore, it can be argued about whether it is a necessary requirement to fully replicate the surface condition in order to understand the interaction between the shoe/foot and the surface. There is also the question of whether the additional surface embedded onto the force platform will influence the ground reaction force data or not. Furthermore, it is unclear if there are any differences in the type of reaction adjustment or alteration performed by human when executing different movement tasks on the same surface prepared in a laboratory-based experiment set up.

Typically, the GRF of the heel and forefoot strike were observed in walking and running analysis because the foot-surface contact phase and its impact force peaks information could potentially provide insight related to movement pattern and injuries occurrence $[11,12]$. These vertical GRF data were commonly normalized to the body mass (also known as the division normalization method) when comparison between subjects of different masses were made [13, 14]. Wannop et al. (2012) argued that besides these commonly use normalization method, there might be other method that could potentially is more effective since the relation between the peak force and body mass might not be always linear [15]. On another note, the ratio between the vertical to shear forces were used in determining the coefficient of traction during footsurface interaction [16], indicating that GRF data can be used in many ways in describing human motion.

The aim of this study was to investigate the difference of vertical force exerted during walking and running motion between movement directly on the force plate and movement onto the synthetic surface-embedded force plate. This study also measured the vertical GRF's ratio between heel-fore foot strike to observe the differences that may occur during walking and running.

\section{Method}

\subsection{Participants}

In this study, five male university level futsal players were recruited (Age $23.5 \pm 2$ years old, body mass $58.5 \pm 6.5 \mathrm{~kg}$, height $165 \pm 6 \mathrm{~cm})$. All of these students are active players in competitive futsal competitions with more than 3 years of experience. The exclusion criteria are: 1) history of fracture in any parts within the lower limb area and 2) lower limb ligament injury. All subjects provided their written informed consent prior to the study, in accordance to the research ethical approval obtained from the research ethics committee of the Faculty of Sports Science and Recreation, Universiti Teknologi MARA. Futsal players were recruited in this study because the synthetic surface used in this study is a surface that is typically used by indoor futsal arena.

\subsection{Instrumentation}

An AMTI Force Plate system (sampling rate $1000 \mathrm{~Hz}$ ) was embedded with a synthetic futsal pitch and Vicon Nexus Motion System (sampling rate 250fps) were utilized to collect the kinetic and kinematics data of walking and running tasks performed by the participants. The experiment set-up is shown in Figure 1. Reflective markers (14mm in diameter) were attached onto 15 bony anatomical landmarks of the participants' lower limbs which includes anterior superior iliac spine, sacral, thigh, knee, tibia, calcaneus, ankle and toe. The technical specification of the synthetic futsal surface is shown in Table 1.

\subsection{Procedure}

All participants wore identical futsal shoe model (Admiral indoor futsal shoe-CTL-5IC) as shown in Figure 2 with different size according to individual fitting and were requested to perform walking $(2.5 \pm 0.5 \mathrm{~m} / \mathrm{s})$ and running $(5.0 \pm 0.5 \mathrm{~m} / \mathrm{s})$ movements on force plate platform. In the first setting, the players who performed the tasks were directly in contact with the force platform surface. In the second setting, the players performed the tasks on a force platform surface that was covered with a futsal court material (Figure 3). All participants performed three trials for each condition.

\subsection{Data Analysis}

The amount of vertical ground reaction force measured by the force platform was normalized to the body weight of each participant. Peak heal strike and peak fore foot strike were identified from the ground reaction force curve. Statistical Package for the Social Sciences (SPSS) version 19 was used to statistically analyze the data collected. Data were analyzed using paired-sample t-test at 0.05 significance level to observe the differences between the two types of surface and movement tasks. 


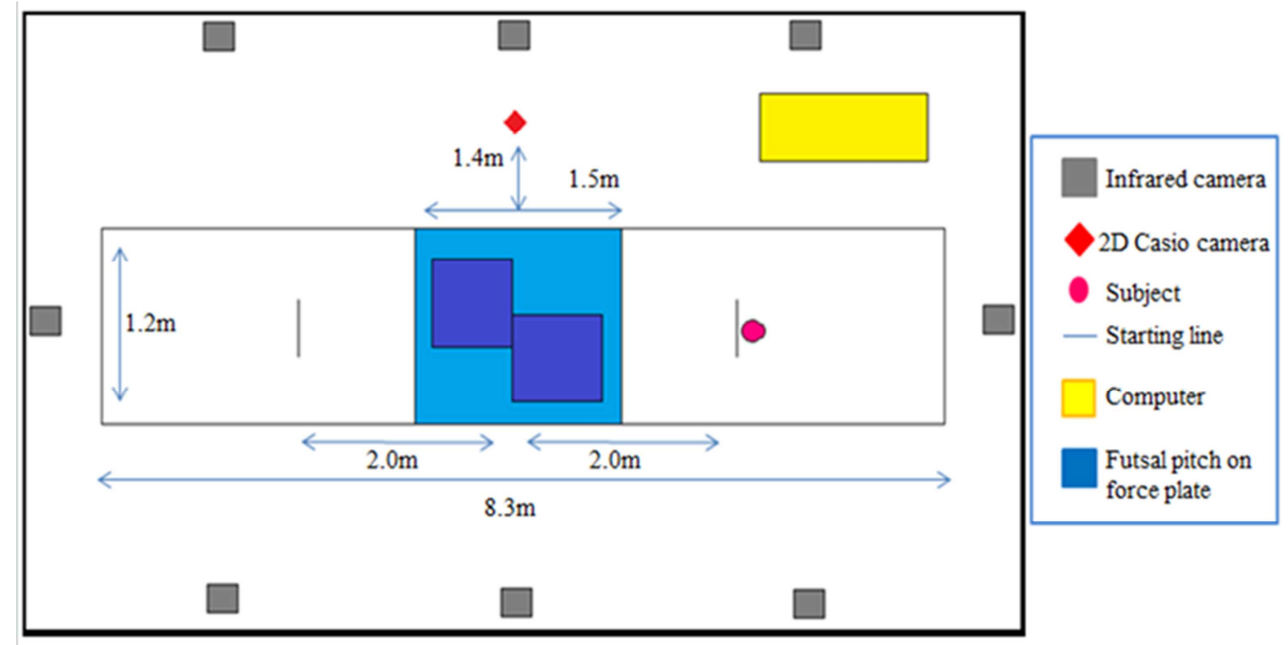

Figure 1. The experiment set-up.

Table 1. Synthetic futsal court material technical specification.

\begin{tabular}{lll}
\hline Description & Material & Polypropylene \\
& Total thickness & $8 \mathrm{~mm}$ \\
Sport characteristics & Shock absorption & $46 \%$ \\
& Sliding coefficient & $0.52-0.6$ \\
Technical & Abrasion resistance & $400 \mathrm{mg}$ \\
characteristics & Impact resistance & $>12 \mathrm{Nm}$ \\
& Residual impression & $0.23 \mathrm{~mm}$ \\
\hline
\end{tabular}

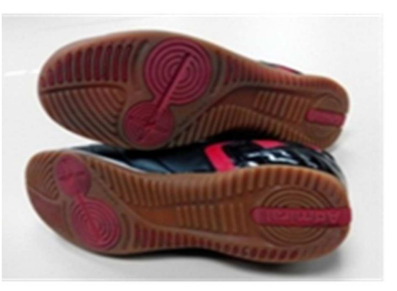

Figure 2. Non-marking outsole footwear.
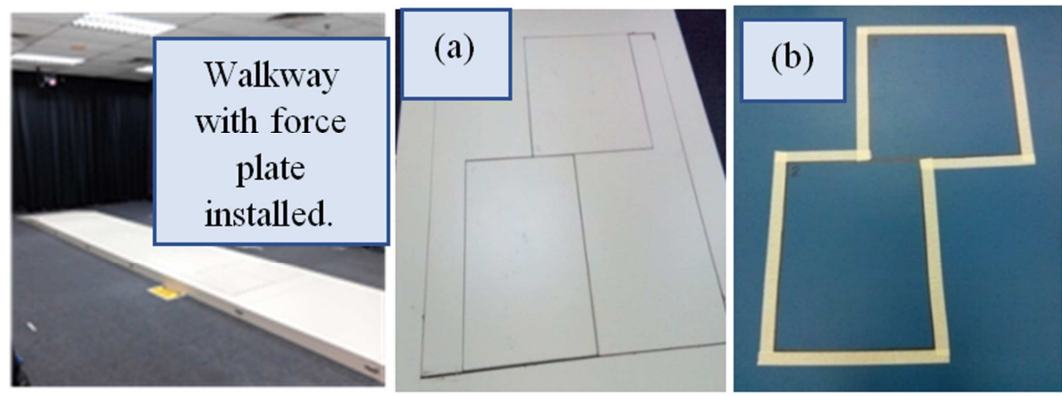

Figure 3. Force platform arrangement: (a) Force platform bare surface, (b) Force platform with futsal court material on top.

\section{Results}

The results obtained from this study are presented in Tables 2, 3 and 4. Table 2 shows the mean peak vertical ground reaction force during heel strike. Table 3 shows the mean peak vertical ground reaction force during forefoot strike. Table 4 explained on the ratio between the mean peak heel-forefoot strike force.

Table 2. Peak vertical ground reaction force during heel strike.

\begin{tabular}{lllllll}
\hline & Type of surface & N (trial) & Mean (SD) & $\boldsymbol{t}$ & $\boldsymbol{d}$ & $\boldsymbol{p}$ value \\
\hline \multirow{2}{*}{ Walking } & Force plate & 15 & $1.16(.08)$ & 1.94 & 28 & .06 \\
& Force plate + futsal court material & 15 & $1.11(.06)$ & & \\
\multirow{2}{*}{ Running } & 15 & $1.27(.36)$ & .45 & 28 & .66 \\
& Force plate & Force plate + futsal court material & 15 & $1.19(.51)$ & & \\
\hline
\end{tabular}

Table 3. Peak vertical ground reaction force during forefoot strike.

\begin{tabular}{lllllll}
\hline & Type of surface & $\mathbf{N}$ (trial) & Mean (SD) & $\boldsymbol{t}$ & $\boldsymbol{d f}$ & $\boldsymbol{p}$ value \\
\hline \multirow{2}{*}{ Walking } & Force plate & 15 & $1.10(.05)$ & -1.31 & 28 & .20 \\
& Force plate + futsal court material & 15 & $1.12(.05)$ & & \\
\multirow{2}{*}{ Running } & Force plate & 15 & $2.16(.11)$ & .42 & 28 & .68 \\
& Force plate + futsal court material & 15 & $2.14(.20)$ & & & \\
\hline
\end{tabular}


Table 4. Peak heel-forefoot strike ratio $(G R F)$.

\begin{tabular}{lllllll}
\hline & Type of surface & $\mathbf{N}$ (trial) & Mean (SD) & $\boldsymbol{t}$ & $\boldsymbol{d}$ & $\boldsymbol{p}$ value \\
\hline \multirow{2}{*}{ Walking } & Force plate & 15 & $1.05(.09)$ & 2.52 & 28 & $.02 *$ \\
& Force plate + futsal court material & 15 & $0.99(.05)$ & & \\
\multirow{2}{*}{ Running } & 15 & $0.61(.18)$ & .94 & 28 & .35 \\
& Force plate & Force plate + futsal court material & 15 & $0.55(.19)$ & & \\
\hline
\end{tabular}

$* \mathrm{p}<0.05$

\section{Discussion}

In this study, the peak vertical ground reaction force during walking and running in two conditions were observed. In the first setting, the participants' performed the tasks directly in contact with the force plate surface. In the second setting, the players performed the tasks on a force plate surface that was covered with a futsal court material. The results indicated that there were no significant differences were in terms of the magnitude of peak heel strike and the peak forefoot strike forces during walking and running, whereby they were with or without the additional futsal court surface on the force platform. Based on the outcomes of this study, we found that adding an additional surface on top of the force platform, in this case a material that is commonly used for a futsal court, will not significantly change the peak vertical ground reaction force of human locomotion in walking and running motions.

These results did not undermine the importance of replicating the exact surface interaction condition of the real condition for laboratory-based force platform setting and experimental setup. However, in a worst-case scenario where the real surface condition was not able to be replicated in the laboratory, a bare-force plate is still useful to understand the kinetics component of a certain movement task. One of the possible way to determine the requirement to replicate the exact same surface interaction between the real condition and laboratory analysis, or not to, is by knowing the range of the ground reaction force magnitudes of the movement to be analyzed. This is because, under high vertical loading, the adhesion friction element dominates the two surfaces in contact, whereas low vertical loading is dominated by hysteretic friction element [17]. This information might be important when considering a new experiment design which requires force plate application. As what was found in this study, during walking (low vertical loading), the aspects of surface material have influenced the peak heel-forefoot strike ratio. The aspects of the surface material hysteretic element may account for it.

Further analysis was performed on the collected data to discover the ratio of the heel-forefoot strike during all tasks. Results shows that there was a significant difference between the ratios of the heel-fore foot strike during walking directly on force platform surface and walking on the force platform with additional surface on it. While the data obtained in this study suggest that an additional surface on the force platform has no significant effect on the magnitudes of ground reaction force data, changes did occur on the heel/forefoot strike force ratio in walking task. This is an indication that the heel-forefoot strike force ratio is a useful indicator that can be used to differentiate movement patterns in human motion study. The result also suggests that an additional surface on the force platform has significant impact on the movement pattern of the foot during walking task due to the different condition of the shoe outsole-flooring surface interaction. These finding shows that different movement mechanism has different interaction aspects even when interacting on the same surface.

In a recent walking gait study [18], it was found that humans tend to alter their body-weight transfer during swing to the support phase when initiating a gait cycle i.e., when walking on different surfaces, particularly on a relatively soft one. This could potentially have influenced the heel-forefoot strike pattern during walking on different foot-surface interaction condition. Since the ground contact time during running can be assumed to be shorter than walking, the same situation might not be able to be observed clearly during running task.

Furthermore, past studies have shown that humans alter their legs' stiffness [7] and adjust their foot-surface traction component [19] to obtain the desired traction condition when running on different surfaces to maintain the running mechanics. Past study also highlighted that humans maintain their running kinematics under different surfaces by adapting their muscle activation to the surfaces [20].

It must be noted here though, that all the results in the present study need to be interpreted with cautious such that in this study, the walking and running velocities of each participant were limited only to certain ranges [ walking $(2.5$ $\pm 0.5 \mathrm{~m} / \mathrm{s})$ and running $(5.0 \pm 0.5 \mathrm{~m} / \mathrm{s})]$. In different human locomotion such as jumping for example, a different outcome might be obtained. Different velocity and vertical loading ranges during locomotion could potentially provide different outcome as well. Furthermore, in the present study, only one type of surface material was tested. A different material with different technical specifications could potentially provide a different outcome. On a different note, in this study, the role of the footwear was not investigated. This is another important aspect in sports surface study because different types of shoe outsoles can influence the footwear and playing surface interactions, specifically in futsal [21]. The cleat designs and material mechanical properties of the shoe and the playing surface aspects could potentially influence sports performance and risk of injury as well $[22,23]$.

\section{Conclusions}

In this study, it was found that additional material on the 
force platform will not significantly influence the peak vertical ground reaction force in walking and running tasks [walking $(2.5 \pm 0.5 \mathrm{~m} / \mathrm{s})$ and running $(5.0 \pm 0.5 \mathrm{~m} / \mathrm{s})$ ] However, it was found that there was a significant difference on the peak heel-forefoot strike ratio during walking task on different foot-surface interactions. This indicates that in a slower velocity movement, the movement pattern changes were observed, potentially due to longer ground contact time during walking gait initiation. No significant changes were observed on the peak heel-forefoot strike ratio during running task, potentially due to the adjustment mechanism by humans to maintain their running mechanics when running on different surfaces. This study also demonstrated that the ratio between the heel and forefoot strike force is a useful parameter in analyzing human motion.

\section{References}

[1] Lorini, G., Bossi, D., Specchia, N., 1992. The concept of movement prior to Giovanni Alfonso Borelli, 23.

[2] Andriacchi, T. P. and Alexander, E. J. (2000). Studies of human locomotion: past, present and future. Journal of Biomechanics, 33, pp. 1217-1224.

[3] Grillner, S., Halbertsma, J., Nilsson, J. and Thorstensson, A. (1979). The adaptation to speed in human locomotion. Brain Res 165, 177-182.

[4] Thorstensson, A., Carlson, H., Zomlefer, M. R. and Nilsson, J. (1982). Lumbar back muscle activity in relation to trunk movements during locomotion in man. Acta Physiol Scand $131,211-214$.

[5] Thorstensson, A., Nilsson, J., Carlson, H. and Zoomlefer, M. (1984). Trunk movements in human locomotion. Acta Ph, ysiol Scand 121, 9-22.

[6] Nilsson, J., Thorstensson, A. and Halbertsma, J. (1985a). Changes in leg movements and muscle activity with speed of locomotion and mode of progression in humans. Acta Physiol Scand 123, 457-475.

[7] Ferris, D. P., Louie, M. and Farley, C. T. (1998) Running in the real world: adjusting leg stiffness for different surfaces. Proc R Soc Lond B Biol Sci 265:989-994.

[8] Cross, R. (1998). Standing, walking, running and jumping on a force plate. American Association of Physics Teachers, Vol. 67, No. 4.

[9] Kirtley, C. The origin of ground reaction forces, [On-line]. Clinical Gait Analysis. Available: www.clinicalgaitanalysis.com (Accessed on August 31, 2017).

[10] Logan, S., Hunter, I., Hopkins, J. T., Feland, J. B. and Parcell, A. C. (2010). Ground reaction force differences between running shoes, racing flats, and distance spikes in runners.
Journal of Sports Science and Medicine, 9: 147-153.

[11] James, S. J., Bates, B. T. and Osternig, L. R. (1978) Injuries to runners. Am. J. Sports Med. 6, 40-50.

[12] Clement. D. B., Taunton. J. E., Smart, G. W. and Nicol. K. L. (1981) A survey of ovcrusc running injuries. Phys. Sportsmed. 9(5), 47-58.

[13] Bates, B. T., Osternig, L. R., Sawhill, J. A., and Hamill, J. (1983). Identification of critical variables describing ground reaction forces during running. In: $\mathrm{H}$. Matsui and $\mathrm{K}$. Kobayashi (eds.), Biomechanics VIII B, International Series on Biomechanics. Champaign, IL: Human kinetics Publishers, 4B, 635-640.

[14] Mullineaux, D. R., Milner, C. E., Davis, I. S., and Hamill, J. (2006). Normalization of ground reaction forces. Journal of Applied Biomechanics, 22, 230-233.

[15] J. W. Wannop, J. T. Worobets, D. J. Stefanyshyn. Normalization of ground reaction forces, joint moments and free moments in human locomotion. J Appl Biomech, 28 (2012), pp. 665-676.

[16] Worsfold P., Smith N., Dyson R. (2007) A comparison of golf shoe designs highlights greater ground reaction forces with shorter irons. Journal of Sports Science and Medicine, 6, 484-489.

[17] Ura D, Conway J, Booth J, Carré M. Tennis shoe outsole temperature changes during hard court sliding and their effects on friction behaviour. Proc Eng. 2015; 112: p. 290-295.

[18] Vieira, M. F., Sacco Ide, C., Nora, F. G., Rosenbaum, D. and Lobo da Costa, P. H. (2015). Footwear and foam surface alter gait initiation of typical subjects. PLoS One. 2015; 10:e0135821. doi:10.1371/journal.pone.0135821

[19] McGhie, D., and Ettema, G. (2013). Biomechanical analysis of traction at the shoe-surface interface on third-generation artificial turf. Sports Engineering 16(2), 71-80.

[20] Dolenec, A., Štirn, I., and Strojnik, V. (2015). Activation pattern of lower leg muscles in running on asphalt, gravel and grass. Collegium Antropologicum, 39(Suppl 1), 167-172.

[21] SI Ismail, H Nunome, FF Marzuki, I Su'aidi. (2018). Measurement of Interaction between Futsal Footwear and Futsal Pitch Surface under Different Outsole Condition. Proceedings 2018, 2(6), 233; doi:10.3390/proceedings 2060233

[22] Diogo C. F. Silva, Rubim Santos, João Paulo Vilas-Boas, Rui Macedo, António Mesquita Montes, and Andreia S. P. Sousa, "Influence of Cleats-Surface Interaction on the Performance and Risk of Injury in Soccer: A Systematic Review," Applied Bionics and Biomechanics, vol. 2017, Article ID 1305479, 15 pages, 2017. doi:10.1155/2017/1305479

[23] Thomson, A., Rod Whiteley, R., \& Bleakley, C. (2015). Higher shoe-surface interaction is associated with doubling of lower extremity injury risk in football codes: A systematic review and meta-analysis. British Journal of Sports Medicine, $49,1245-1252$. 